\title{
Identification of $\beta$-Haemolytic Streptococci among Pharyngitis Cases at Bir Hospital, Kathmandu
}

\author{
A. K. Shrestha ${ }^{1}$, B. Lekhak ${ }^{1}$, J. Shrestha ${ }^{2}$ and D. R. Bhatta ${ }^{1}$ \\ ${ }^{1}$ Central Department of Microbiology, Tribhuvan University, Kathmandu \\ ${ }^{2}$ Pathology Department, Bir Hospital, Kathmandu \\ e-mail: atmazshrestha@gmail.com
}

\begin{abstract}
Respiratory tract infection is one of the major reasons of patients seeking medical care. Among infections pharangitis is most common in the upper respiratory tract, which is seen most frequently in children and adolescents and less frequently among adults. The objective of this study was to analyze the underlying bacterial pathogens in pharyngitis cases and antibiotic susceptibility of various isolates. During February to August, 2010, a total of 134 patients visiting E.N.T. OPD of Bir Hospital complaining sore throat were studied. The throat swabs of the patients were cultured in $5.0 \%$ sheep blood agar plates and incubated at $37^{\circ} \mathrm{C}$ for $24-48 \mathrm{hrs}$. Clear transparent $\beta$-haemolytic colonies of the germs were selected and identified. Group A streptococcus was identified by $\beta$-haemolytic colonies with bacitracin and penicillin sensitivity, gram staining, catalase negativity and growth on crystal violet blood agar. In this study, $23(17.2 \%)$ patients were positive for $\beta$-haemolytic streptococci. Among them, $12(9 \%)$ were identified as Group A streptococcus. The culture positive for BHS was highest (56.5\%) among the age group of 21-40 years. Pathogens other than $\beta$-haemolytic streptococci were isolated from 9 patients, of which 4 cases showed mixed bacterial growth. Other bacterial isolates reported in the study were Staphylococcus aureus 6 (4.5\%), Klebsiella pneumoniae $5(3.7 \%)$, Pseudomonas aeruginosa $2(1.5 \%)$, Citrobacter freundii $1(0.7 \%)$, and yeast cells $1(0.7 \%)$. It was found that infections caused by $\beta$-haemolytic streptococci was significantly higher $p=0.0084$ i.e. $(p<0.05)$ than infections caused by other bacteria. This study demonstrated that young adults of age group 21-40 were affected by streptococcal pharyngitis. The isolates of GAS were $100 \%$ sensitive to amoxycillin, cephalexin, cefotaxime, ciprofloxacin and azithromycin followed by cotrimoxazole (91.7\%) and erythromycin (91.7\%).
\end{abstract}

Key words: $\beta$-haemolytic streptococci, bacitracin, group A streptococcus, pharyngitis, sore throat

\section{Introduction}

Streptococcus pyogenes (group A streptococcus, GAS) is an important species of gram-positive bacterial pathogen which colonizes the throat of human beings and causes pharyngitis (Cunningham 2000, Pfoh et al. 2008). Streptococcal pharyngitis continues to be one of the most common childhood illnesses throughout the world (Bisno 1996, Linder et al. 2005). Rheumatic fever $(\mathrm{RF}$ ) is a delayed sequel to GAS pharyngitis (Dale \& Beachey 1986, Adderson et al. 1998).

It is estimated that over 600 million cases of symptomatic GAS pharyngitis occur annually among people aged over 4 years, and that over 550 million of these occur in less developed countries. Additional cases occur in children aged under five years, although GAS pharyngitis becomes less common in younger children. Therefore, it seems reasonable to conclude that approximately $15 \%$ of school-age children suffer symptomatic episode of GAS culture-positive pharyngitis each year in more developed countries, and that $4-10 \%$ of adults are similarly affected. The incidence of pharyngitis cases in less developed countries, may be five to ten times greater than more developed countries on basis of the data from India, Kuwait, the New Zealand, Maori and Pacific Islander populations (Carapetis et al. 2005). To initiate control programme of RF/RHD in less developed countries data on the prevalence and incidence of â-haemolyic streptococci, especially GAS pharyngitis are required. 
However, only scanty information is available in Nepal regarding the magnitude of this problem.

The aim of this study was to explore the current situation of streptococcal infection as well as other bacterial pathogens in the throat of symptomatic patients visiting Bir Hospital. Moreover, to study antibiotic sensitivity pattern of isolated pathogens.

\section{Methodology}

Altogether 134 throat swab samples were processed for culture, isolation and identification of causative organisms of pharyngitis/tonsillitis. Patients with sore throat visiting E.N.T. Out-patient Department of Bir Hospital, recommended by medical practitioners for throat swab culture were included in the study. The throat swabs obtained were cultured in sheep blood agar (BA) and MacConkey agar (MA) plates within 30 minutes upon receipt at the laboratory. Standard protocols of Vandepitte et al. (2003) and Cheesbrough (2006) were followed for culture and identification of bacteria. The cultures inoculated on blood agar plates were incubated at $37^{\circ} \mathrm{C}$ in $5-10 \%$ carbon dioxide and examined after overnight incubation and again after 48 hours. All the plates with â-haemolytic colonies identified by conventional methods (colony morphology, haemolysis pattern, catalase test, gram staining). Streptococcus pyogenes was further identified by observing its sensitivity towards 0.04 units of bacitracin disc and 10 units of penicillin disc on blood agar plate. Isolates from aerobically incubated MacConkey agar plates were identified using colony morphology, gram-staining and biochemical tests. All the pathogenic microorganisms isolated from throat swab samples were subjected for antimicrobial susceptibility test by Kirby-Bauer disk diffusion method as recommended by CLSI. $S$. pyogenes ATCC 19615 was taken as control for susceptibility test of low concentration of bacitracin for identification of GAS. For the standardization of Kirby-Bauer test and for performance testing of antibiotics and MHA, control strains of E. coli (ATCC 25922) and S. aureus (ATCC 25923) were tested primarily. Antibiotics discs containing penicillin 10 units, amoxycillin $10 \mathrm{mcg}$, chloramphenicol $30 \mathrm{mcg}$, ciprofloxacin $5 \mathrm{mcg}$, cotrimoxazole $25 \mathrm{mcg}$, cephalexin $30 \mathrm{mcg}$, cefotaxime $30 \mathrm{mcg}$, erythromycin $15 \mathrm{mcg}$, azithromycin $15 \mathrm{mcg}$, amikacin $30 \mathrm{mcg}$, gentamicin 10 mcg, polymyxin B 300 units were tested for isolates.

\section{Results and Discussion}

Out of 134 samples, $17.2 \%$ (23) were found to be âhaemolytic streptococci (BHS), Bacterial pathogens other than BHS accounted for $6.7 \%(9 / 134)$ cases and no pathogenic organisms were reported in $76.1 \%$ (102/ 134) cases. Group Aâ-haemolytic streptococcus (GAS) constituted $52.2 \%(12)$.

On the basis of agewise distribution of the âhaemolytic streptococci, 56.5\% ( $\mathrm{n}=13)$ BHS were isolated from the age group $21-40$ and $21.8 \%(n=5)$ BHS were isolated from age group of 16-20. Similarly $13 \%(n=3)$ BHS from age group $<15$ and $8.7 \%(n=2)$ BHS from age group of 41-65 were found. There was no significant association between â-haemolytic streptococci cases and the age group of the patients $(p>0.05)$, (Table 1$)$.

Table 1. Age and gender wise distribution of the patients with positive cases of BHS

\begin{tabular}{l|c|c|c|c}
\hline Age group & \multicolumn{2}{|c}{ Number } & Total \\
\hline & Male & Female & 3 & 13.0 \\
\hline 15 & 3 & 0 & 5 & 21.8 \\
$16-20$ & 4 & 1 & 13 & 56.5 \\
$21-40$ & 5 & 8 & 2 & 8.7 \\
Total & 0 & 2 & 23 & 100 \\
\hline
\end{tabular}




\section{Microbiological pattern}

Out of 134 throat swabs that were cultured, 23 BHS were isolated and nine cases showed pathogenic microorganisms other than BHS. Out of nine cases 4

Table 2. Microbiological pattern of throat isolates cases had mixed bacterial growth. The other pathogenic microorganisms isolated were S. aureus 6 (4.5\%), K. pneumoniae 5 (3.7\%), P. aeruginosa 2 $(1.5 \%)$, C. freundii $1(0.7 \%)$, and Yeast Cells. $1(0.7 \%)$, (Table 2).

\begin{tabular}{|c|c|c|c|c|c|c|}
\hline \multirow{2}{*}{ Organisms } & \multicolumn{2}{|c|}{ Male } & \multicolumn{2}{|c|}{ Female } & \multirow[t]{2}{*}{ Total \% } & \multirow{2}{*}{$\begin{array}{l}\text { Total no. } \\
\text { of } \\
\text { isolates }\end{array}$} \\
\hline & $\begin{array}{l}\text { No. of } \\
\text { isolates }\end{array}$ & $(\%)$ & $\begin{array}{l}\text { No. of } \\
\text { isolates }\end{array}$ & $(\%)$ & & \\
\hline$\beta$ Haemolytic streptococci other than Group A & 3 & 2.2 & 8 & 6.0 & 8.2 & 11 \\
\hline Group A $\beta$ Haemolytic streptococcus & 5 & 3.7 & 7 & 5.2 & 9.0 & 12 \\
\hline Staphylococcus aureus & 4 & 3.0 & 2 & 1.5 & 4.5 & 6 \\
\hline Klebsiella pneumoniae & 2 & 1.5 & 3 & 2.2 & 3.7 & 5 \\
\hline Pseudomaonas aeruginosa & 2 & 1.5 & 0 & 0 & 1.5 & 2 \\
\hline Citrobacter freundii & 0 & 0 & 1 & 0.7 & 0.7 & 1 \\
\hline Yeasts & 1 & 0.7 & 0 & 0 & 0.7 & 1 \\
\hline
\end{tabular}

Antibiotic susceptibility test (AST) of the â-haemolytic streptococci isolates

The antibiotic sensitivity pattern of GAS showed that most sensitive drugs for GAS were amoxycillin, cephalexin, cefotaxime, ciprofloxacin and azithromycin, which were $100 \%$ sensitive followed by cotrimoxazole
(91.7\%) and erythromycin (91.7\%). Most sensitive drugs for BHS were amoxycillin, cefotaxime, ciprofloxacin and azithromycin, which were 100\% sensitive followed by cotrimoxazole $(90.9 \%)$, erythromycin $(72.7 \%)$ and cephalexin $(63.6 \%)$, (Table 3).

Table 3. Antibiotic sensitivity pattern of â-haemolytic streptococci isolates

\begin{tabular}{|c|c|c|c|c|c|c|c|c|c|c|}
\hline \multirow[t]{3}{*}{ Antibiotics } & \multicolumn{5}{|c|}{ BHS Other than Group A } & \multicolumn{5}{|c|}{ GAS } \\
\hline & \multicolumn{2}{|c|}{ Sensitive } & \multirow{2}{*}{$\begin{array}{r}\text { Intermediate } \\
\text { No. }\end{array}$} & \multicolumn{2}{|c|}{ Resistant } & \multicolumn{2}{|c|}{ Sensitive } & \multirow{2}{*}{$\begin{array}{c}\text { Intermediate } \\
\text { No. }\end{array}$} & \multicolumn{2}{|c|}{ Resistant } \\
\hline & No. & $\%$ & & No. & $\%$ & No. & $\%$ & & No. & $\%$ \\
\hline Amoxycillin & 11 & 100 & 0 & 0 & 0 & 12 & 100 & 0 & 0 & 0 \\
\hline Cephalexin & 7 & 63.6 & 0 & 4 & 36.4 & 12 & 100 & 0 & 0 & 0 \\
\hline Cefotaxime & 11 & 100 & 0 & 0 & 0 & 12 & 100 & 0 & 0 & 0 \\
\hline Cotrimoxazole & 10 & 90.9 & 0 & 1 & 9.1 & 11 & 91.7 & 0 & 1 & 8.3 \\
\hline Ciprofloxacin & 11 & 100 & 0 & 0 & 0 & 12 & 100 & 0 & 0 & 0 \\
\hline Erythromycin & 8 & 72.7 & 0 & 3 & 27.3 & 11 & 91.7 & 0 & 1 & 8.3 \\
\hline Azithromycin & 11 & 100 & 0 & 0 & 0 & 12 & 100 & 0 & 0 & 0 \\
\hline
\end{tabular}




\section{Antibiotic sensitivity pattern of bacteria other than â-haemolytic streptococci isolates}

The antibiotic sensitivity pattern of bacterial isolates other than BHS showed highest sensitivity to ciprofloxacin (78.6\%), gentamycin (75\%) and amikacin
(75\%), followed by chloramphenicol (71.4\%), cefotaxime $(57.1 \%)$, azithromycin $(66.7 \%)$, penicillin $(33.3 \%)$ and cephalexin $(42.9 \%)$ and least sensitivity to amoxycillin (28.6\%) and cotrimoxazole $(21.4 \%)$. Polymyxin B was used only for P. aeruginosa, (Table 4).

Table 4. Antibiogram sensitivity pattern of bacteria other than â-haemolytic streptococci isolates

\begin{tabular}{l|c|c|c|c|c|c|c}
\hline \multicolumn{1}{c|}{ Antibiotics } & \multicolumn{2}{c|}{ Sensitive } & \multicolumn{2}{c|}{ Resistant } & Total \\
\hline & No. & $\%$ & No. & $\%$ & No. & $\%$ & \\
\hline Ciprofloxacin & 11 & 78.6 & 0 & 0 & 3 & 21.4 & 14 \\
Amoxycillin & 4 & 28.6 & 1 & 7.1 & 9 & 64.3 & 14 \\
Cephalexin & 6 & 42.9 & 1 & 7.1 & 7 & 50.0 & 14 \\
Cefotaxime & 8 & 57.1 & 0 & 0 & 6 & 42.9 & 14 \\
Gentamicin & 6 & 75 & 0 & 0 & 2 & 25.0 & 8 \\
Amikacin & 6 & 75 & 0 & 0 & 2 & 25.0 & 8 \\
Chloramphe ricol & 10 & 71.4 & 0 & 0 & 4 & 28.6 & 14 \\
Cotrimoxazole & 3 & 21.4 & 0 & 0 & 11 & 78.6 & 14 \\
Polymin B & 2 & 100 & 0 & 0 & 0 & 0.0 & 2 \\
Azithromycin & 4 & 66.7 & 0 & 0 & 2 & 33.3 & 6 \\
Pericillin & 2 & 33.3 & 0 & 0 & 4 & 66.7 & 6 \\
\hline
\end{tabular}

In this study, 134 throat swab samples were processed for detection of bacterial pathogens causing pharyngitis. Out of $23(17.1 \%)$ cases that were found to be positive to BHS and 12 cases were accounted for GAS. This study showed that the prevalence of GAS was $9 \%$ in patients. Similar studies conducted by Bista et al. (2005), Shrestha et al. (2006), Rijal et al. (2009) and Gurung et al. (2010) showed the prevalence of GAS being $18 \%, 58.8 \%, 9.2 \%$ and $5 \%$ respectively. However, in the present study more samples were obtained from adults of age $>15$ years. Only Bista et al. (2005) in above mentioned study included adult population. In this study among 23 positive âhaemolytic cases, $88.46 \%$ (20/23) patients were of $>15$ years of age. Data obtained in this study correlate with the studies of Poses et al. (1985), Komaroff et al. (1986) and Ebell et al. (2000) that the prevalence of GAS was reported to be around $5 \%$ to $10 \%$ in adults. The high prevalence of BHS in adult population in patients in our study may be due to hygienic and socioeconomic conditions the adult population serves as carrier of BHS. In this study, the frequency of BHS was not similar in all age groups. It was slightly higher in adults aged $21-40$ years, however, it was statically insignificant $(p>0.05)$. In this study it has been seen that the age of the patients coming for treatment shifted from school aged children to young adults (21-40 years of age). This is more likely because Bir Hospital entraps more of adult population and children that usually visit children's hospital located nearby, thus eluding attention.

In this study highest number of pathogenic organisms in throat swab was represented by BHS, then followed by S. aureus $6(4.5 \%), K$. pneumoniae $5(3.7 \%), P$. aeruginosa $2(1.5 \%)$, C. freundii $1(0.7 \%)$ and yeast 1 $(0.7 \%)$ respectively (Table 2$)$. According to Vandepitte et al. (2003) these microorganisms do not cause pharyngitis, except in association with granulocytopenia. It is advisable to report such isolates to the clinicians, as they occasionally indicate the existence of (or may sometimes give rise to) a lower respiratory tract infection (e.g. pneumonia) or bacteraemia. Isolation of these organisms in the throat 
may be due to transient colonisation of these organisms in adults secondary to repeated use of antibiotics (Longanathan et al. 2006). In this study it was found that infections caused by BHS in tonsillitis/pharyngitis patients is significantly higher than infections caused by other pathogenic bacteria ( $p=0.0084$ i.e. $p<0.05$ ). Thus, according to the data obtained it can be said that BHS were the major pathogens in pharyngitis cases.

In present study, S. aureus and K. pneumoniae were reported in $4.5 \%$ and $3.7 \%$ cases respectively. Bista et al. (2005) in comparative study of core and surface culture of tonsillectomy cases in Tribhuvan University Teaching Hospital (T.U.T.H.) reported S. aureus in $21 \%$ of cases. Likewise, K. pneumoniae on $10 \%$ cases, both organisms were more significantly seen in $>20$ age group than others. According to Longanathan et al. (2006), S. aureus was reported in $45.5 \%$ of cases and was the most common organism in adults with sore throat followed by K. pneumonia in $26.0 \%$ of cases, and was the second most common organism. The low prevalence of these organisms in the present study may be due to pharyngeal swab cultures which do not reliably reflect the presence of pathogens in the tonsilar core as shown in the study carried out by Al-roosan et al. (2008).

It was observed that most sensitive drugs for GAS were amoxycillin, cephalexin and cefotaxime, ciprofloxacin and azithromycin, which were 100\% sensitive, followed by cotrimoxazole $(91.7 \%)$ and erythromycin (91.7\%). The most sensitive drugs for BHS other than Group A are amoxycillin, ciprofloxacin and azithromycin, which were $100 \%$ sensitive. The result of sensitivity pattern of macrolide used in present study correlates with study carried out by Rijal et al. (2009), which showed $97.8 \%$ isolates were sensitive to azithromycin and $84.4 \%$ were sensitive to erythromycin. Dumre et al. (2009) reported 94.8\% isolates were sensitive to erythromycin. According to Gurung et al. (2010) erythromycin showed $100 \%$ sensitivity. The present study also revealed that macrolide antibiotics were sensitive towards GAS. Hence, azithromycin is a drug of choice for pharyngitis patients, who are allergic to penicillin.

In the present study, GAS isolates showed $91.7 \%$ sensitivity to ciprofloxacin and cotrimoxazole. This finding is in agreement with that of Dumre et al. (2009), which reported $94.8 \%$ isolates sensitive to ciprofloxacin but $29.0 \%$ were sensitive to cotrimoxazole.

In this study amoxycillin inhibited $80 \%$ of S. aureus, cotrimoxazole inhibited only $40 \%$ of the isolates whereas azithromycin and penicillin inhibited $80 \%$ and $60 \%$ of isolates respectively. In the study carried out by Shrestha et al. (2006), amoxycillin and cotrimoxazole inhibited $76.3 \%$ of isolates of S. aureus, erythromycin and penicillin inhibited $65.8 \%$ and $44.7 \%$ isolates, respectively.

The results showed that BHS was one of the major causes of bacterial pharyngitis among adult population. It has been demonstrated that antibiotic resistance of $S$. pyogenes is not a clinically significant problem. The results of this study also highlighted the importance of regular programs of monitoring the rate of GAS carriage and the antibiotic susceptibility of GAS isolates in adult population.

In the treatment of clinical pharyngitis, it is known that oral antibiotics do not give as much coverage as benzyl penicillin to patients who may later go on to develop RF/RHD due to lack of adequate antibiotic protection. However, over treatment with penicillin may pave way to increased penicillin resistance. Finally, one may encounter larger number penicillin anaphylaxis, which can significantly reduce patient's compliance to antibiotic treatment. Thus, scientifically, it is better to manage treatment after confirming a GAS etiology which would overcome the above problems (Gurung et al. 2010).

This study concludes that information of GAS susceptibility pattern is crucial for monitoring the development of antibiotic resistance in S. pyogenes. During this study, $76.1 \%$ culture results showed the growth of non pathogenic microorganisms, which may be either due to viral infection (Cheesbrough 2006) in patients or allergic reactions. Immunohistochemical findings Modrzyñski et al. (2003) hypothesized that allergic sensitization takes place in the adenoid and tonsils. Further, Modrzyñski et al. (2005) confirmed that tonsil dendritic cells, macrophages, eosinophils, and mast cells are important in allergic tonsillitis. Therefore, viruses along with quantitative analysis of bacterial molecular biology together with allergic reactions in consideration will be necessary in the future research. 
Nepal Journal of Science and Technology Vol. 14, No. 2 (2013) 137-142

Our present findings demonstrated that young adults (age group: 21-40) are affected by BHS tonsillitis/ pharyngitis, so further long term study is needed in this matter.

\section{Acknowledgments}

The authors would like to acknowledge all the staff and management committees of the Bir hospital as well as the faculty and staff of Central Department of Microbiology, Kirtipur for their kind cooperation during the study period.

\section{References}

Adderson, E.E., A.R. Shikhman, K.E. Ward and M.W. Cunningham. 1998. Molecular analysis of polyreactive monoclonal antibodies from rheumatic carditis: human anti-N-acetylglucosamine/antimyosin antibody $\mathrm{V}$ region genes. Journal of Immunology 61: 2020-2031.

Al-roosan, M.,N. Al-khtoum and H. Al-said. 2008. Correlation between surface swab culture and tonsillar core culture in patients with recurrent tonsillitis. Khartoum Medical Journal 01: 129-132.

Bisno, A.L. 1996. Acute pharyngitis: etiology and diagnosis. Pediatrics 97: 949-954.

Bista, M., B.K. Sinha, R.C.M. Amatya, N.R. Tuladhar and B.M. Pokharel. 2005. Comparison of core and surface cultures in recurrent tonsillitis. Journal of Institute of Medicine 27: 60-65.

Carapetis, J.R., A.C. Steer, E.K. Mulholland and M. Weber. 2005. The global burden of group A streptococcal diseases. Lancet Infectious Disease 5: 685-694.

Cheesbrough, M. 2006. District laboratory practice in tropical countries. 2nd ed. Cambridge University Press.

Cunningham, M.W. 2000. Pathogenesis of group A streptococcal infections. Clinical Microbiology Reviews 13: 470-511.

Dale, J.B. and E.H. Beachey. 1986. Sequence of myosincrossreactive epitopes of streptococcal $\mathrm{m}$ protein. Journal of Experimental Medicine 164: 1785-1790.

Dumre, S.P., K. Sapkota, N. Adhikari, D. Acharya, M. Karki, S. Bista and S.R. Basanyat. 2009. Asymptomatic throat carriage rate and antimicrobial resistance pattern of Streptococcus pyogenes in Nepalese school children. Kathmandu University Medical Journal 7: 392-396.

Ebell, M.H., M.A. Smith, H.C. Barry, K. Ivesand M. Carey. 2000. The rational clinical examination. Does this patient have strep throat? Journal of the American Medical Association 284: 2912-2918.
Gurung, R., S. Budhathoki, R. Amatya, N. Poudyal, S. Shrestha and R. Baral. 2010. Group A â-haemolytic streptococcus infection presenting with sore throat at paediatric OPD. Health Renaissance 8: 90-92.

Komaroff, A. L., T.M. Pass, M.D. Aronson, C.T. Ervin, S. Cretin, R.N. Winickoff, W.T Branch, and B. Brigham. 1986. The prediction of streptococcal pharyngitis in adults. Journal of General Internal Medicine 1: 1-7.

Linder, J.A., D.W. Bates, G.M. Lee and J.A. Finkelstein. 2005. Antibiotic treatment of children with sore throat. Journal of American Medical Association 294: 2315-2322.

Longanathan, A., U.D. Arumainathan and R. Raman. 2006. Comparative study of bacteriology in recurrent tonsillitis among children and adults. Singapore Medical Journal 47: 271-275.

Modrzyñski, M., P. Grochowski, E. Zawisza and A. Lipiec. 2003. Histopathological and immunological analysis of hyperplastic palatine tonsils and adenoids in children with coexisting atopic dermatitis. Przegl Lek 60: $317-321$.

Modrzyñski, M., H. Mazurek and E. Zawisza. 2005. Allergic tonsillitis: myth or reality. Postepy Hig Med Dosw 59: 450-456.

Pfoh, E., M.R Wessels, D. Goldmann and G.M. Lee. 2008. Burden and economic cost of group A streptococcal pharyngitis. Pediatrics 121: 229-34.

Poses, R.M., R.D. Cebul, M. Collins and S.S. Fager. 1985. The accuracy of experienced physicians' probability estimates for patients with sore throats: implications for decision making. Journal of American Medical Association 254: 925-929.

Rijal, K.R., N. Dhakal, R.C. Shah, S. Timilsina, P. Mahato, S. Thapa and P. Ghimire. 2009. Antibiotic susceptibility of group A streptococcus isolated from throat swab culture of school children in Pokhara, Nepal. Nepal Medical College Journal 11: 238-240.

Shrestha, A., J.B. Sherchand, P.R. Sharma and D.R. Bhatta. 2006. Bacteriological study of upper respiratory tract infection in pediatric patients. Journal of Institute of Medicine 28: 28-31.

Somro, A., M. Akram, I.M. Khan, H.M. Asif, A. Sami, S.M.A. Shahi, G. Shahen, K. Ahmed and R.U. Rehman. 2011. Pharyngitis and sore throat: A review. African Journal of Biotechnology 10: 6190-6197.

Stillerman, M. and S. Bernstein. 1961. Streptococcal pharyngitis: evaluation of clinical syndromes in diagnosis. American Journal of Diseases of Children 101: 476-489.

Vandepitte, J., J. Verhaegen, K. Engbaek, P. Rohner and C.C. Heuck. 2003. Basic laboratory procedures in clinical bacteriology. 2nd ed. Geneva: World Health Organization. 